\title{
Cuestiones filosóficas de la pedagogía en las ciencias de la educación en Francia
}

Armando Zambrano Leal ${ }^{1} \mathbb{O}$

\section{@() $\Theta \Theta$}

\footnotetext{
1. Secretaría de educación Valle del Cauca, Colombia.

azambranoleal@gmail.com
}

Recibido: 05/09/2021

Revisado: 08/10/2021

Aprobado: 10/11/2021

Publicado: 01/01/2022

\section{Resumen}

Las ciencias de la educación en Francia son una disciplina universitaria de saber plural, cuyo objeto de estudio son el acto y el hecho educativos. Esta disciplina nació en 1967 y se ha consolidado como el espacio de referencia de investigación y formación en dicha sociedad. En el ámbito de las ciencias de la educación, la pedagogía abarca un conjunto de discursos y de prácticas dirigidas a la comprensión de la acción de educar y se nutre de las cuestiones filosóficas vitales frente a la instrumentación del acto educativo. Nuestro propósito, en este artículo, es mostrar algunos resultados de nuestras reflexiones sobre la disciplina y, en especial, sobre las cuestiones y los conceptos de la filosofía de la educación en la pedagogía.

Palabras clave: ciencias de la educación, filosofía de la educación, pedagogía, pedagogos, conceptos 


\title{
Philosophical issues of pedagogy in educational sciences in France
}

\begin{abstract}
In France, educational sciences are an academic field of plural knowledge, focused on the educational act and the educational fact. This discipline was established in 1967 and has become the reference point for research and training in that society. Within educational sciences, pedagogy encompasses a set of discourses and practices aimed at understanding the action of educating and is nourished by crucial philosophical questions regarding the implementation of the educational act. Our purpose, in this article, is to present some results of our reflections on this discipline and, in particular, on the issues and concepts of philosophy of education in pedagogy.
\end{abstract}

Key words: educational sciences, philosophy of education, pedagogy, pedagogues, concepts

\section{Questões filosóficas da pedagogia nas ciências da educação na França}

\section{Resumo}

Na França, as ciências educacionais são um campo acadêmico de conhecimento plural, focado no ato e no fato educacionais. Esta disciplina nasceu em 1967 e se tornou o ponto de referência para a pesquisa e a formação naquela sociedade. Dentro das ciências da educação, a pedagogia engloba um conjunto de discursos e práticas destinadas a compreender a ação de educar e é alimentada por questões filosóficas fundamentais relativas à instrumentação do ato educacional. Nosso objetivo, neste artigo, é apresentar alguns resultados de nossas reflexões sobre esta disciplina e, em particular, sobre as questões e conceitos de filosofia da educação em pedagogia.

Palavras-chave: ciências da educação, filosofia da educação, pedagogia, pedagogos, conceitos 
Como resultado de la larga historia sobre la formación de una disciplina para la República, las ciencias de la educación en Francia se institucionalizaron a través del acto administrativo del 7 de diciembre de 1967, expedido durante la reforma de la enseñanza superior según el decreto del 11 de febrero de 1967 (Zambrano, 2013, p. 100). Como disciplina universitaria, tienen por objeto la investigación, la formación y la transmisión de los saberes relacionados con el acto y hecho educativos. Este objeto es plural y significa que diversas disciplinas se ocupan de su comprensión y de su explicación, pero también de las acciones prácticas. Mientras el acto educativo es objeto de la sociología, de la antropología, de la psicología, de la pedagogía, de la didáctica y de la filosofía, el hecho educativo es más del ámbito de la historia y de ciertos trabajos de orden sociológico.

Se entiende el acto educativo como la acción presente por medio de la cual la transmisión del saber, la experiencia humana acumulada, los principios y valores, la cultura y una visión e idea de ser humano se actualizan en la acción escolar y familiar. El hecho educativo, por su parte, se comprende como el efecto de la acción sobre las personas, las instituciones y la sociedad (Zambrano, 2006a).

Así mismo, el acto y el hecho educativos se materializan, respectivamente, en el tiempo presente y pasado: lo que se enseña y lo enseñado, lo aprendido, lo instituido y lo instituyente, los programas y sus transformaciones, etc. La organización escolar, por ejemplo, es un fuerte componente de la historia de la educación, así como también de las formas escolares que investiga la sociología de la educación (Charlot, 2001; Lahire, 2005; Perrenoud, 2005).

Dos grandes saberes tienen lugar en las ciencias de la educación: de un lado, está la didáctica, cuya disciplina estudia la génesis, la circulación y la apropiación del saber escolar; y, de otro lado, la pedagogía, cuyo discurso se dirige a las cuestiones filosóficas que emergen en el acto de educar (Zambrano, 2005, 2006b, 2016). Estos dos saberes se nutren de disciplinas, saberes y discursos distintos. Mientras la didáctica ha consolidado su objeto gracias a los aportes de la psicosociología, la antropología, el psicoanálisis y la epistemología (Zambrano, 2006c); la pedagogía lo hace desde la filosofía, en particular. De manera preponderante, la pedagogía se ha ocupado de la enseñanza y reflexiona los fines y finalidades de la acción educativa (Zambrano, 2019). De ahí provienen los conceptos ilustrados y contemporáneos como la libertad, la autonomía, la otredad, sujeto, poder, etc.

Esto ha sido muy importante en las ciencias de la educación y tiene su origen en fundamentos discursivos cuyos referentes encontramos en la historia de las doctrinas pedagógicas. En especial, los pedagogos franceses han bebido de la cultura alemana y de la tradición filosófica francesa. Así mismo, se han nutrido de las diferentes orientaciones filosóficas de los grandes pedagogos. Por ejemplo, los pedagogos médicos en el siglo XIX se dieron a la tarea de reeducar el lenguaje, trabajaron arduamente en esta perspectiva y acudieron a Herbart y Pestalozzi, entre otros. Jean Marc Gaspard Itard y Éduard Seguin trabajaron, entre otros aspectos, la reeducación moral y fisiológica del sordo. Un concepto central de estos dos grandes pedagogos médicos fue la formación intelectual y del espíritu. Las sensaciones, el medio cultural y las conductas fueron conceptos claves en la formación moral del niño con necesidades educativas especiales. De Itard, por ejemplo, se conoce el gran trabajo sobre Victor d'Aveyron, El niño 
salvaje; de Éduard Seguin, una importante obra sobre el idiotismo y otras discapacidades (Truffaut, 1970). Se sabe, por ejemplo, que la pedagoga italiana María Montessori se nutrió de los presupuestos filosóficos y de las técnicas de estos dos pedagogos médicos. Así mismo, ellos inspiraron toda la corriente de la pedagogía experimental observada, entre otros, en las investigaciones de Alfred Binet.

Así, entonces, la pedagogía refleja, trae y se nutre de la filosofía. De allí nacen las grandes cuestiones sobre la educación del sujeto y sobre los fines para la sociedad. Esta característica muestra un registro poco explorado en las comprensiones sobre ella. En otras palabras, conocer las cuestiones filosóficas de la pedagogía en el ámbito de las ciencias de la educación permite entender por qué los pedagogos más representativos de la disciplina trascienden el instrumento técnico para pensar filosóficamente las cuestiones vivas de la acción de educar. Indiscutiblemente, este asunto nos lleva a otra gran pregunta, que consiste en saber quién es pedagogo. Por el momento, afirmo que pedagogo es aquella persona cuya práctica la conduce a pensar filosóficamente las cuestiones inherentes a la educación de alguien.

\section{Movimientos pedagógicos y conceptos antes de las ciencias de la educación}

Para comprender el lugar de la pedagogía en el seno de las ciencias de la educación, es importante identificar los momentos y movimientos que se gestaron antes y durante la institucionalización de la disciplina. Indiscutiblemente, la educación nueva -o pedagogía moderna - introduce una nueva perspectiva. Se trató del lugar del niño en la educación escolar. En el caso francés, luego de terminada la Primera Guerra Mundial, los pedagogos comenzaron a reactivar los aportes de los pedagogos médicos y se dieron a la tarea de relacionar el concepto ilustrado de libertad con el de educabilidad. Estos dos conceptos fueron importantes en el nacimiento de la pedagogía experimental en la década de 1930.

Se le debe a Edmund Demolin, fundador de la Escuela de Roches en 1898 en Verneuilsur-Avre, la introducción y fuertes cambios en las formas de organización de la enseñanza, la implementación de los métodos activos, la observación del trabajo escolar y la puesta en funcionamiento de los centros de interés forjados por Decroly. En definitiva, la educación nueva - tal como se conoce en los trabajos de Adolphe Ferrière y otros- parte del hecho de que, para lograr la libertad en el niño, debe partirse de su capacidad para ser educado educabilidad-, de la atención, de su propia actividad, de las necesidades según su edad, de sus gestos e intereses personales y de la reacción contra los métodos tradicionales (Foulquié, 1971, p. 159).

La educación nueva se comprende como "el conjunto de proposiciones y de acciones originales constituyéndose como corriente innovadora hacia finales del siglo XIX y una oposición fundadora respecto a la pedagogía tradicional" (Morandi, 1997, p. 49). Como elementos fundamentales para la pedagogía, la educación nueva se inspiró en la tradición empirista de Condillac, Rousseau y Voltaire y en el positivismo de Spencer, Comte, Durkheim. También integró el existencialismo y el pragmatismo, lo que muestra una fuerte y estrecha relación con el liberalismo. 
Los principios de la educación nueva están plasmados en la Liga Internacional para la Educación Nueva, cuya adopción tuvo lugar en 1921. Sin lugar a duda, el concepto central de la educación nueva fue el de activo. Este concepto tiene tres momentos sucesivos: elaboración entre 1910 y 1920, el de la Arbeitsschule — cuyo creador fue Georg Kerschensteiner- y la escuela del trabajo de Adolphe Ferrière -entre 1917-1920- (Hameline et al., 1995). El planteamiento central, el que observamos en los pedagogos activos, consiste en poner en relación la escuela con la vida. De allí surgieron diferentes métodos. Este gran movimiento introdujo la cooperación en la escuela gracias a la práctica del pedagogo francés Celestin Freinet (1969), quien establece 24 principios para la educación del niño, para su libertad y para su formación como ciudadano.

La educación nueva engendró el movimiento conocido como pedagogía nueva o pedagogía activa. El concepto de nuevo presupone la ruptura con las prácticas cerradas y ancianas heredadas del catolicismo pedagógico. Este concepto recogió una nueva lectura de libertad. El niño es libre en su acción y su educación reafirmará en su espíritu esta necesidad de desarrollar sus capacidades para decidir por sí mismo. La formación del juicio, su capacidad de conocimiento, la disposición para encauzar sus disposiciones y la creatividad estuvieron ligadas al desarrollo de métodos que las permitieran. En este orden, la pedagogía moderna se preguntó por la educación del niño, por los fines y la naturaleza de la educación, por los medios que la hacen posible, por la relación con el mundo y por su constitución como sujeto. La cuestión central, sin duda alguna, fue la libertad, pues el fondo del asunto, proveniente de las luchas sociales, fue la formación de la ciudadanía. Por lo tanto, activo y libertad fueron fines de la educación para una sociedad en plena transformación. De allí nacieron los métodos activos, los que, a la vez, observamos en los pedagogos como Freinet, Ferrière, Decroly, etc.

Como resultado de la pedagogía moderna, antes de la institucionalización de las ciencias dela educación, germinó la pedagogía experimental —o científica-. El presupuesto filosófico parte del hecho de que lo activo y la libertad están lejos de cualquier espontaneidad. Esta pedagogía reconoce el lugar del niño en la institución y propone investigar las diferentes problemáticas que allí se presentan. Relaciona laboratorio con ciencia y es altamente positivista. Considera que el saber activo es productor de métodos, pero ello exige deducir los comportamientos a fin de poder mejorar los métodos y extraer de esta experiencia el saber formador de los maestros. Es en esta práctica donde nacen las escuelas-taller. Sus resultados fueron claves para la formación de los maestros en las escuelas normales.

Los trabajos de Alfred Binet y Théodore Simon muestran la fuerte presencia de la psicología del desarrollo de la inteligencia. Este movimiento buscó responder a las preguntas: ¿la actividad del niño y los programas propuestos responden a los fundamentos de la libertad? ¿Es el niño capaz por sí solo de dotarse de valores y juicios capaces de conducirlo a su libertad? Para responder a dichas preguntas, se recurrió al experimento, a la observación de los métodos, a la medición de resultados, a la definición de nociones claras y precisas enseñables a través de los programas, a las dificultades tanto en los maestros como en los estudiantes y al análisis de las habilidades, destrezas y capacidades para el trabajo en grupo y autónomo. Estas líneas de acción reflejaban una variante de la educación nueva, pues no se podía responder a la libertad 
sin antes explicarlas, sin observarlas, sin obtener conclusiones que permitieran mejorar las acciones escolares. El movimiento no tuvo mayores desarrollos en Francia y terminó refundándose en otro gran movimiento conocido como la psicopedagogía.

Esta buscó llevar a la práctica los métodos de la pedagogía experimental y, para ello, acudió al saber de la psicología y a la pedagogía como aplicación. Se proponía ser reconocida como la ciencia de la educación, instancia formadora y productora de saber (Mialaret, 1986). Este saber se refiere a las formas escolares de constitución y emancipación del sujeto y por ello se nutrió del psicoanálisis y de los postulados de la educación nueva. Para este movimiento, la educación no podía considerarse como "perfeccionamiento de las facultades humanas, sino como el conjunto de influencias del medio ordenadas en relación con la maduración biológica las cuales permiten el desarrollo del niño" (Hameline, 1979, p. 16). Este movimiento logró crear una serie de institutos en algunas universidades, pero pronto fueron cerrados.

Luego de la psicopedagogía, emergió un movimiento muy importante, conocido como pedagogía institucional. Nació del seno de la educación nueva en la corriente de Celestin Freinet. Contrario a la clase cooperativa, la pedagogía institucional es una confluencia del marxismo, el campo terapéutico y el psicoanálisis. Trabaja los conceptos de institución, psicoterapia y análisis. Para esta corriente, la pedagogía es un dispositivo de análisis de la institución, de los sujetos, del aula de clase y del malestar en relación con lo instituido e instituyente. Curiosamente, el movimiento tiene fuertes raíces en las prácticas del Hospital Psiquiátrico de Saint Alban y en las nuevas perspectivas sobre la cura. La conciencia del enfermo y su libertad para decidir cuándo salir y cuándo entrar fueron retomadas por los psiquiatras Fernand Oury y Félix Guattari en la clínica de La Borde. Ellos traen al campo de la educación los conceptos del derecho a la locura, oposición contra el encierro y el trabajo en el reconocimiento del otro. Se nutrieron, igualmente, del psicoanálisis de Lacan y de la fenomenología de Sartre y Merleau-Ponty.

Fernando Oury retomó el concepto de pedagogía institucional, forjado por su hermano Jean Oury. El sujeto niño es analizado a partir de la crítica a la institución y a la autogestión. Luego, Georges Lapassade retomó la teoría crítica de la sociedad y de la institución en dos importantes libros de Henry Lefevbre, Crítica de la razón dialéctica y Crítica del concepto de grupo. Con ellos analizó, a partir de los conceptos de política y psicosociología, el tema de la burocracia en la institución escolar (Hess, 1976). A este trabajo se unieron Remi Hess, Michel Lobrot, René Lourau, Patrice Ville y Gérard Althabe. Este grupo inició un trabajo importante sobre el análisis de grupo-clase, la autogestión de la clase y las relaciones no directivas (Schaepelynck, 2012).

La cuestión filosófica de este movimiento está dirigida a la emancipación de los sujetos a través de la autogestión. La pedagogía, en este sentido, es un discurso instituyente de emancipación y un espacio de contrainstitucionalidad. La libertad del sujeto es el resultado de la autoconciencia respecto de su condición de esclavitud. Ella ve en los procesos instituyentes formas de enajenación del sujeto y medios de fabricación de su subjetividad. La autogestión está fuertemente ligada a la autonomía y a la consciencia del sujeto, dos mecanismos de libertad.

Estos movimientos muestran una línea de lucha y de pensamiento a través de la interrogación sobre los conceptos de activo, experimento e institución. También visibilizan la preocupación 
por la educación del niño, sus finalidades y su naturaleza. Para la educación nueva, es el niño en su subjetividad quien marca las acciones de la educación. Es su naturaleza y su especificidad la que muestra el camino de los métodos. Por su parte, la experimentación —científicaintentó adentrase en el alma de los sujetos, de la institución y de los programas, para deducir los cambios y ajustes de la educación para la ciudadanía y para su libertad.

En cuanto ala institución, queda claro que el tema dela autogestión es fuente de emancipación, pues las luchas del sujeto están dirigidas a toda forma de opresión instituida y, por esta vía, a la imposible libertad gestionada desde la propia institución. Estos movimientos dejan clara una lectura distinta de la pedagogía, pues ella es algo más que una simple técnica para la enseñanza. En estos movimientos, y especialmente en la educación nueva y en la pedagogía institucional, se observa una lectura crítica respecto de la pedagogía oficial, reduccionista y alineada a los objetivos. Esto encuentra mayor desarrollo en el paso de la pedagogía por objetivos, la cual fue adoptada oficialmente por el Estado tres años después de institucionalizadas las ciencias de la educación, a la pedagogía diferenciada. Esta última introduce el concepto de diferencia para avanzar más en los análisis de los conceptos de activo, institución y grupo. Louis Legrand (1983, p. 283) fue el primer pedagogo en advertir el hecho de que lo activo no podía homogenizarse, tampoco la institución ni el grupo, sin antes detenerse en la diferencia constitutiva de cada uno. Él se apoyó en los trabajos de Gilles Deleuze, especialmente en su libro Diferencia y repetición (1968). La diferencia es tanto sociocultural, de valor, creencias, historia familiar, códigos de lenguaje y tipos de socialización. También se observa lo psicoafectivo en la cognición y sus tipos (Fournier, 1996, p. 117).

Es claro, entonces, que los movimientos pedagógicos anteriores a la constitución de las ciencias de la educación se apoyan en conceptos filosóficos y, a partir de ellos, se cuestionan los fines y la naturaleza de la educación. Estos movimientos fueron claves en la disciplina, pues muchos pedagogos contemporáneos retoman los conceptos para argumentar sus visiones sobre la pedagogía.

\section{La pedagogía en las ciencias de la educación}

Una vez constituidas e institucionalizadas las ciencias de la educación, muchos teóricos de los movimientos pedagógicos ingresaron a la disciplina. Desde allí, iniciaron un trabajo de discusión sobre el qué de la pedagogía y su condición plural. Cada uno expresó su definición según el momento y la época. Por esto, tal vez sería más conveniente hablar de pensamiento pedagógico, pues la pedagogía no es la misma en todas las épocas. Se desarrolla en los momentos críticos de la historia de la sociedad. Es una especulación normativa que gira en torno al avenir, porque entiende lo que "debe hacerse" y es una necesidad permanente en la sociedad moderna (Kerlan, 2001). En general,

ella es portadora de un discurso de legitimización, vía la práctica y está vinculada con el pensamiento educativo de una época. Dos herencias marcan el principio de definición: el racionalismo universalista y el del naturalismo evolucionista del siglo XIX. El primero centra su proyecto educativo sobre la autonomía y la razón y el progreso intelectual. El segundo lo hace sobre la autonomía de la acción y el perfeccionamiento moral. (Bulle, 2005, p. 117) 
La fuerte presencia de la psicología incitaba a algunos pedagogos a considerar que ella no era por sí misma la fuente de inspiración de la pedagogía. Por ejemplo, Chateau (1967) afirmaba:

Así como sucede con el médico frente a la cura de la enfermedad, no se pueden esperar los conocimientos biológicos o los medicamentos aún desconocidos, el educador no puede esperar a la psicología... es necesario que el genio individual supla las carencias de la pedagogía. (p. 10)

En relación con la práctica y la teoría, la pedagogía plantea la acción reflexiva. "Ella es la acción que efectúa el pedagogo sobre sí mismo" (Houssaje, 1984, p. 31). Para Mialaret (1999), uno de los fundadores más importantes de las ciencias de la educación, hay que entender la pedagogía en sus raíces: el esclavo que acompaña al hijo del amo hasta la entrada al liceo. En la modernidad, especialmente para los movimientos pedagógicos posteriores al surgimiento de la educación nueva, "ella está relacionada con una finalidad, una disciplina y las capacidades" (p. 31).

Para Avanzini (1992),

la pedagogía responde a los procesos normativos y prescriptivos. La primera, prescribe la conducta a partir de principios, propone doctrinas y la segunda, es productora de teorías. El proceso normativo tiene tres orígenes: la filosofía, la teología y la política. El proceso prescriptivo, por su parte, emerge de las ciencias, teorías y prácticas de observación. (p. 16)

Según Meirieu (1997), "la pedagogía es la inteligencia del acto educativo, especialmente porque la educación es una relación asimétrica, necesaria y provisional que apunta a la emergencia del sujeto" (p. 23).

Según Beillerot (1983),

la pedagogía cuyo objeto es el saber de una sociedad y, su finalidad, el proceso de influencia por medio del proceso enseñar-aprender podría entenderse como una relación estudianteprofesor (poder), un discurso sobre lo real que se dice o no del saber o del conocimiento, saber que no es solamente del profesor o del libro; un conjunto de prácticas que explican (actualización de regularidades) para hacer comprender (percibir las significaciones) interpretar (organizarla en conceptos) y verificar. (p.10)

Para Filloux (1983), la pedagogía es una práctica de iniciación:

Poder conocer el deseo del pedagogo es deseo del placer de engendrar y una lectura psicoanalítica de los mitos y fantasmas permite restaurar como constitutivo de la posición del pedagogo en el campo escolar... la escena significativa de la relación pedagógica; aquella de un deseo por engendrar; deseo de una procreación y deseo de un nacimiento. Pero se trata, claro está, de un segundo nacimiento como nacimiento del espíritu y de una segunda procreación en tanto creación cultural. (p. 18)

Estas y otras definiciones resaltan la pluralidad de perspectivas y más aún cuando se trata de su lugar - ¿colonización o dominación? - en las ciencias de la educación, en primer lugar, porque la disciplina recoge las diferentes corrientes de pensamiento; en segundo lugar, porque cada pedagogo expresa su experiencia, convicción y práctica; y en tercer lugar, porque detrás de cada definición hay una visión de la educación concomitante con la época, con la fuerza de los 
ideales, con las luchas y con las creencias sobre el porvenir de la educación. Tal vez, el consenso es más visible a la hora de ponerla en relación con el acto educativo, pues este supone "un conjunto coherente de acciones emprendidas con miras a alcanzar un fin, un sistema ordenado de medios y porque es la puesta en obra de principios implícitos y explícitos provenientes de una teoría general" (Postic, 1986, pp. 19-20).

La filosofía de la educación y los pedagogos franceses que de ella se nutren abarcan tres grandes momentos: el de finales del siglo XIX, donde se multiplican los cursos de educación, pedagogía o psicología de la educación y cuyos precursores fueron, por lo general, filósofos; un segundo momento, en la segunda mitad del siglo XX, está fuertemente unido a la participación de filósofos en la psicopedagogía, especialmente en las escuelas normales; y un tercer momento, el de la institucionalización de las ciencias de la educación, ve florecer a un grupo de filósofos de la educación que inician un largo camino de reflexión sobre la naturaleza de la pedagogía y de la educación (Kahn, 2006).

\section{Algunos conceptos filosóficos en la pedagogía}

Hay acuerdo entre los pedagogos franceses sobre las finalidades de la pedagogía. No hay duda de que ella interroga el provenir de lo humano en la figura del niño o del joven. Esto tiene una fuerte relación con la educación como proceso liberador, como acción de valor y como momento del ser. Educar es la finalidad humana suprema ya como perfectibilidad, ya como inacabamiento, ya como reactivación de la experiencia humana en el tiempo. O mejor, la pedagogía interroga al ser humano en su devenir esencial (Soëtard, 2001, p. 10).

Aquí resaltan dos grandes conceptos: el de tradicional y el de nuevo. Mientras para la educación tradicional el sistema metódico impone la fuerte presencia del saber adulto; en la educación nueva, es la naturaleza del niño la que marca el camino (Bérengère, 2018). Esto tiene su anclaje en la tradición del concepto de activo, fuertemente trabajado en los pedagogos de la educación nueva. Este concepto es central en las diferentes pedagogías, por ejemplo, en la institucional y la diferenciada. La finalidad de la educación - escolar- no se reduce a la transmisión de un saber dado, institucionalizado, operado por las ciencias, instituido por las disciplinas. Va más allá y se interroga la libertad del otro encarnada en la figura del sujeto estudiante.

Cabe aquí distinguir entre finalidad y fin de la educación (Dupuis, 2002). Si la educación es formar el hombre en el hombre, su fin es la conciencia de un espíritu cuyas capacidades lo llevan a gobernarse. La finalidad se inscribe en la libertad y en cuanto hace y actúa. Al mismo tiempo, es inherente a un interior reflexivo, su fin último, como obra de sí mismo. El fin de la educación, dice Reboul (1971, p. 25), es permitirle a cada uno cumplir su naturaleza en el seno de una cultura que sea verdaderamente humana. Hay libertad y su dilucidación compromete a quienes, al reflexionar sobre la acción educativa, ven en ella un momento para sí mismos. La libertad, entonces queda expuesta a las diversas reflexiones que los pedagogos realizan y a los fines del método.

Si la finalidad es la libertad reflexionada, entonces se impone la relación medios-fines y sobre ella giran, entre otros conceptos, los de otro, valor, ética, institución, ciudadanía, saber y 
moral. Por ejemplo, el lugar de la ética en las disquisiciones de los pedagogos siempre habitará como cuidado e implica responsabilidad. Mientras la ética alude al cuestionamiento sobre lo que es justo y bueno hacer, la moral exige la obediencia a lo exigido y la sumisión de nuestra naturaleza sensible a la naturaleza racional, a los imperativos de la razón práctica (Kernan, 2003, p. 74) si la libertad aparece como fin de la acción educativa.

La constelación de conceptos advierte, en la obra de los pedagogos franceses inscritos en las ciencias de la educación, una fuerte interrogación en el siguiente orden. El sujeto es la finalidad de la acción educadora. Durante el proceso, él es otro cuya raíz encontramos en los trabajos de la filosofía de la otredad. Por ejemplo, Meirieu (1991), apoyado en Levinas, intenta responder a la cuestión por el otro en su libertad constitutiva (Zambrano, 2009). Así mismo, Kerlan (1998) reflexiona sobre la ética del cuidado del otro en la lectura sobre Paul Ricoeur. De igual manera, a Poizat (2011) la obra de Levinas y su concepto de otro - autrui- le permiten comprender la relación ética y de libertad en la acción educativa. Pero este otro también aparece en la figura del maestro, en su condición de transmisor y de puente entre las generaciones. Si se habla de otro, no queda duda del lugar preponderante de la alteridad en la relación maestro-alumno (Hansel, 2011). Esta imagen del maestro es recurrente en cuanto pasador de experiencia y saber, como otro en su libertad constitutiva, como portador de una historia y de filiación en el nacimiento de autrui, el alumno (Perrin, 2009).

Si el concepto de otro es recurrente en los pedagogos, también lo son los de valor, sujeto, autoridad, nacimiento, institución y saber. Estos y otros conceptos giran en las discusiones sobre el fin y la finalidad de la pedagogía. En cuanto a su fin, ella se preocupa por la comprensión de la acción tanto en la figura del alumno como del profesor. El terreno es la educación escolar. En cuanto a su finalidad, ella sobrepasa los métodos, pues provee una comprensión de la acción educativa siempre en relación con la libertad. Esta es la gran diferencia en relación con aquellos discursos prácticos y técnicos que ven en la pedagogía un instrumento organizador de los saberes, pero también de las formas de ser estudiante - obediencia, disciplina, autoridad, sumisión, etc-. La pedagogía en la tradición de las ciencias de la educación es un ámbito de reflexión hermenéutica o fenomenológica, en particular.

\section{Las grandes cuestiones}

A pesar de las fuertes reticencias respecto de los filósofos de la educación en las discusiones pedagógicas (Moreau, 2010), en el seno de las ciencias de la educación emerge un movimiento de la filosofía de la educación agrupado en la Sociedad Francófona de Filosofía de la Educación (Sofphied) (Houssaye, 2009). Existen, así mismo, algunas revistas como Le Telémaque y la publicación de la Asociación de Profesores de Ciencias de la Educación, quienes mantienen en sus congresos y coloquios una línea especial para los filósofos de la educación. Diversas revistas de filosofía acogen las reflexiones sobre la educación de quienes hacen obra pedagógica. La Sofphied está integrada por pedagogos y filósofos interesados en las cuestiones filosóficas sobre la educación. En su creación, la cuestión de partida consistió en debatir la existencia o no de la filosofía de la educación como área de estudio y de formación y su relación con la pedagogía, con la educación y con la formación.

En lo estrictamente pedagógico, los filósofos de la educación se interrogan: ¿qué saberes 
hay que enseñar? ¿Qué valores y qué fines? ¿Qué es la autoridad? ¿Qué es ser maestro? ¿Es necesario suspender la libertad o esperarla? Un segundo bloque de cuestiones está relacionado con la práctica pedagógica. Ella no se limita al conjunto de técnicas o a su racionalidad. Va más allá, pues interroga la acción del maestro como productor de saber pedagógico reflexivo. ¿La acción pedagógica del maestro es portadora de saber? Entonces, ¿de qué naturaleza es dicho saber? Un tercer bloque es visible en la actualización de los grandes filósofos y su papel en las comprensiones del hecho pedagógico. ¿Qué papel juegan los grandes filósofos en la reflexión educativa? ¿Cómo traer al plano de la pedagogía el pensamiento de, por ejemplo, filósofos como Alain, Spencer, Platón, Aristóteles, Pestalozzi, Rousseau, Levinas y Ricoeur, entre otros? ¿Cómo leer la acción educativa desde el pensamiento del filósofo y por qué? Un cuarto bloque se pregunta por la naturaleza de la pedagogía en la relación constitutiva y de emergencia del sujeto. La interrogación mayor está relacionada aquí con el poder liberador del saber y de la consciencia del sujeto en la sociedad. ¿La educación domestica o libera? El papel liberador del maestro no consiste en la réplica de los métodos ni en la sumisión frente a los saberes prescritos. Su capacidad de invención y de juego frente al poder es fuente de resistencia. El maestro al enseñar se libera tanto de sus prejuicios como de la obediencia implantada a lo largo de sus años de formación. Sin embargo, tal vez la cuestión crucial de la filosofía de la educación en la pedagogía consiste en saber a qué llamamos pedagogía. ¿Es ella una acción y de qué tipo? ¿Su estatus es decisivo para los fines y finalidades de la educación? ¿Qué se juega cuando un maestro y un estudiante, en su constitución como sujetos, entran en relación de saber?

En fin, los pedagogos franceses, aquellos que hacen de la acción una ocasión para reflexionar los fines y finalidades de la acción educativa, tienen la gran virtud de volver su mirada sobre la filosofía y ven lo que la técnica oculta, aquello que esconde el poder prescriptor de los saberes oficiales, las trampas del saber institucionalizado y el saber escolar vehiculizado en las políticas y programas. La acción pedagógica es reflexión sobre lo humano en lo humano, es una poética del momento engendrador de libertad. Por esto, para el pedagogo nunca será suficiente con advertir el sinuoso poder de los medios y las técnicas. Él va más allá e interroga el valor de educar. En últimas, el pedagogo, al nutrirse de la filosofía de la educación, se pregunta qué es educar, qué es formar. Los pedagogos franceses son hijos de la Ilustración, pero también de Grecia y hasta del estoicismo. Las distintas escuelas filosóficas los acompañan en la bella acción de pensar la libertad educadora. Los pedagogos franceses ven en la filosofía lo que la psicología o la sociología ocultan.

\section{Referencias}

Avanzini, G. (1992). Introduction aux Sciences de l'Education. Privat.

Beillerot, J. (1983). Contribution à l’analyse de la pédagogie. Revue Française de Pédagogie, (64), 7-22.

Bérengère, K. (2018). Louis Legrand philosophe et léducation nouvelle. Les Sciences de l'éducation - Pour l'Ère nouvelle, 51(2), 73-89.

Bulle, N. (2005). La pensé pédagogique moderne : entre science et politique. En D. Kamboucher, \& T. Jacques-Francillon (Eds.), La crise de la culture scolaire (pp. 185-189). PUF. 
Charlot, B. (2001). Le rapport au savoir en milieu populaire. Une recherche dans les Lycées professionnels de banlieue. Anthropos.

Chatêau, J. (1967). Pour une éducation scientifique. Revue Française de Pédagogie, (1), 9-16.

Deleuze, G. (1968). Diferencia y repetición. AMORRORTU.

Dupuis, P. (2002). Qu'est-ce que la pédagogie ? Le pédagogue au risque de la philosophie [compterendu]. Revue Française de Pédagogie, (139), 171-173.

Filloux, J. (1983). Clinique et pédagogie. Revue Française de Pédagogie, (64), 13-20.

Foulquié, P. (1971). Dictionnaire de la langue pédagogique. PUF.

Fournier, M. (1996). La pédagogie différencié. En M. Fournier (Coord.), Eduquer et former. Sciences Humaines.

Freinet, C. (1969). Les dits de Mathieu. Delachaux et Niestlé.

Hameline, D. (1979). Intégration sociales et accomplissement de soi dans la psychologie moderne. En D. Hameline, J. Houssaye, P. Kaeppelin, P. Mayol, J. Milet, J. Piveteau, P. Rey-Herme, \& D. Dubarle, Le sujet de l'éducation. Beauchesne.

Hameline, D., Janoud, A., \& Belkaïd, M. (1995). L’École Active. Textes fondateurs. PUF.

Hansel, J. (2011). Éthique et enseignement. La figure du maître dans totalité et infini. Les Temps Modernes, (664), 151-169.

Hess, R. (1976). La pédagogie institutionnelle. Éditions Universitaires.

Houssaye, J. (1984). L’esclave pédagogue et ses dialogues. Education et Recherche, (1), 31-48.

Houssaye, J. (2009). De la naissance des philosophes de l'éducation en France. En A. Vergnioux (Dir.), 40 ans des sciences de l'éducation (pp. 165-178). PUC.

Kahn, P. (2006). La philosophie de l’éducation au miroir de l’histoire. Le Télémaque, (30), 131-136.

Kerlan, A. (1998). La Science néduquera pas. Peter Lang.

Kerlan, A. (2001). À quoi pensent les pédagogues ? La pensée pédagogique au miroir du philosophe. Revue Française de Pédagogie, (137), 17-26.

Kerlan, A. (2003). Philosophie pour l'éducation. ESF.

Lahire, B. (2005). L'invention de l'Illettrisme. La Découverte; Poche.

Legrand, L. (1983). Pour un collège plus démocratique. Rapport au Ministre de l'Éducation National. La Documentation Française.

Meirieu, P. (1991). Le choix d’éduquer. Éthique et pédagogie. ESF.

Meirieu, P. (1997). Praxis pédagogique et pensée de la pédagogie. Revue Française de Pédagogie, (120), 25-37.

Mialaret, G. (1986). La psychopédagogie. PUF.

Mialaret, G. (1999). Pédagogie générale. PUF.

Morandi, F. (1997). Modèles et méthodes en pédagogie. Nathan.

Moreau, D. (2010). Philosophie et pédagogie : Dilthey au secours de Pestalozzi, ou l'effort herméneutique 
pour comprendre l'éducation [Ponencia]. Congrès International Actualité de la Recherche en Education et en Formation, AREF, Ginebra, Suiza.

Perrenoud, P. (2005). Métier délève et sens du travail scolaire (6a ed.). ESF.

Perrin, C. (2009). Enseigneur et Maître : Heidegger pédagogue. Revue Philosophique de la France et l'Étranger, (134), 333-354.

Poizat, D. (2011). Présentation et lecture de : Il nous manque une culture. Réflexions sur l'enseignement hébraïque d’Emmanuel Levinas. Le Télémaque, (40), 105-118.

Postic, M. (1986). La relation éducative. PUF.

Reboul, O. (1971). La philosophie de léducation. PUF.

Schaepelynck, V. (2012). Emancipación y relación pedagógica en el análisis institucional. En A. Zambrano, P. Meirieu, E. Theodoropoulou, A. Mutuale, \& V. Schaepelynk, Filosofía de la educación y pedagogía. Editorial Brujas.

Soëtard, M. (2001). Qu’est-ce que la pédagogie ? Le pédagogue au risque de la philosophie. PUF.

Truffaut, F. (1970). L'enfant sauvage [película]. Les Films du Carrosse, Les Productions Artistes Associés.

Zambrano, A. (2005). Didáctica, pedagogía y saber. Magisterio.

Zambrano, A. (2006a). Las ciencias de la educación en Francia: relación intrínseca e histórica del acto educativo. Educere, 10(34), 415-420.

Zambrano, A. (2006b). Las ciencias de la educación y didáctica: hermenéutica de una relación culturalmente específica. Educere, 10(35), 593-599.

Zambrano, A. (2006c). Los hilos de la palabra. Pedagogía y didáctica. Magisterio.

Zambrano, A. (2009). El mínimo gesto: la cuestión de la ética en el pensamiento pedagógico de Philippe Meirieu. Revista Educación y Pedagogía, 12(28), 25-37.

Zambrano, A. (2013). Las ciencias de la educación en Francia. Instituciones, movimientos y discursos. Magisterio.

Zambrano, A. (2016). Pedagogía y didáctica: esbozo de las diferencias, tensiones y relaciones de dos campos. Praxis \& Saber, 7(13), 45-61.

Zambrano, A. (2019). Naturaleza y diferenciación del saber pedagógico y didáctico. Pedagogía y Saberes, (50), 75-84. 